\title{
Potential model for tetrathiafulvalene based on inelastic neutron scattering and Raman spectra
}

\author{
Gary R. Burns \\ School of Chemical and Physical Sciences, Victoria University of Wellington, P.O. Box 600, Wellington, \\ New Zealand \\ Frederic Favier, Deborah J. Jones, and Jacques Rozière \\ Laboratoire des Agrégats Moléculaires et Matériaux Inorganiques (LAMMI), ESA 5072 CNRS, Université \\ Montpellier 2, Place Eugène Bataillon, 34095 Montpellier Cédex 5, France \\ Gordon J. Kearley \\ Interfacultair Reactor Institute, Mekelweg 15, 2629JB Delft, The Netherlands
}

(Received 18 November 2002; accepted 3 June 2003)

\begin{abstract}
Tetrathiafulvalene (TTF) is a key molecule in the family of charge-transfer salts of interest for their novel transport properties. The correspondence between transport property and electron-molecular vibration coupling requires that we understand the phonon density of states for these materials. Here we report the results of a low temperature study of neutral TTF using both inelastic neutron scattering spectroscopy and Raman spectroscopy. The new data has been interpreted using the CLIMAX code to calculate the neutron spectral profile which has also been calculated based on a set of force constants derived using Density Functional Theory to calculate the ground state energy of neutral TTF. The two approaches lead to a concordant set of harmonic force constants for the in-plane and out-of-plane normal modes of TTF. (C) 2003 American Institute of Physics.
\end{abstract}

[DOI: $10.1063 / 1.1594719]$

\section{INTRODUCTION}

Determining the electron-donor properties of organic molecules such as tetrathiafulvalene (TTF) and bis(ethylenedithio)tetrathiafulvalene (BEDT-TTF) is central to achieving an understanding of the transport properties of their salts, including those that are superconducting. It is important that the degree of partial electron transfer from TTF be determined as this correlates with the electronic conductivity of the salt. It has been argued that this conductivity is strongly dependent on the strength of electron-phonon coupling ${ }^{1-3}$ and hence knowledge of the vibrational and electronic energies of both the neutral parent molecules and of their salts is necessary in order to explain and predict the transport properties.

The transport and mechanical properties of conducting salts modified by intercalation in suitable lamellar hosts are of interest as a source of hybrid materials for applications as sensors and nonlinear optical devices. ${ }^{4,5}$ The intercalation of a variety of TTF cations into $\gamma$-ZrPhosphate $(\gamma$-ZrP) (Ref. 6) and $\mathrm{MnSP}_{3}$ (Ref. 7) are examples of such hybrid materials. Similar mechanisms are expected to apply to the transport properties of intercalated salts and hence there is a need to extend the study of the vibrational energy states to these systems. More recently, there has been a focus on the synthesis of substituted TTF's for application as cation sensors, liquid crystals, intramolecular charge-transfer, and nonlinear optical materials and supramolecular switches. ${ }^{8}$ Here also the key to understanding the role of the electronic structure of the substituted TTF is to have data on the vibrational and electronic energy states.

It is significant that no complete analyses of the vibra- tional states for either BEDT-TTF or TTF have yet been reported. Eldridge et al. ${ }^{9}$ have studied the room temperature infrared and Raman spectra of BEDT-TTF and four of its isotopic analogs and report a modified valence force model that successfully predicts most of the in-plane and a few of the ethylene out-of-plane modes. The work of Bozio et al. ${ }^{10,11}$ remains the definitive infrared and Raman study of TTF and TTF ${ }^{1.0}$ and their force fields satisfactorily explain most of the in-plane modes. More recently, Liu et al. ${ }^{12}$ have used a combination of density functional theory and $a b$ initio MP2 calculations to study the structures and vibrational spectra of both BEDT-TTF and TTF. However, while the understanding of the in-plane modes for both $\mathrm{TTF}_{-} \mathrm{H}_{4}$ and TTF- $\mathrm{D}_{4}$ is good there is limited data for the out-of-plane modes and therefore the potential models for TTF and BEDT-TTF remain incomplete. The difficulty of determining a complete potential model for TTF is in large part due to the low infrared and Raman activity of these out-of-plane modes, and to the photosensitivity of the crystals which has hindered the measurement of polarization data. Here we report the results of studies of the vibrational energy states of crystalline TTF at low temperatures, using a combination of inelastic neutron scattering (INS) spectroscopy and Raman spectroscopy.

The advantage of INS for the study of molecules containing hydrogen atoms is the enormous neutron scattering cross section for protons when compared to any other isotope, coupled with the strong dependence of the INS intensity on the RMS displacement of atoms in a normal coordinate. The low energy and relatively large displacements of the out-of-plane modes involving protons ensures that they 


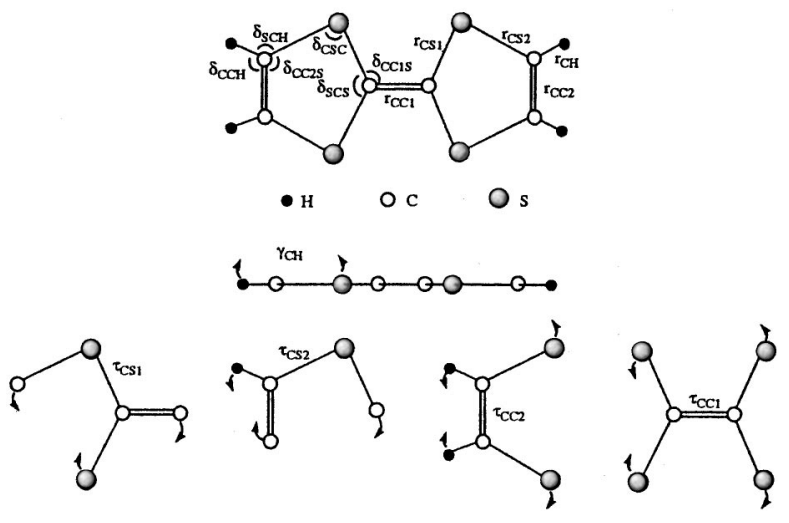

FIG. 1. The molecular structure and coordinate definitions for TTF.

contribute significant intensity in the INS spectrum. Here we report the measurement of INS and low temperature Raman scattering spectra of TTF to determine the out-of-plane modes. Under the low temperature conditions of the INS and Raman experiments TTF is stable and we report good quality spectra for the neutral TTF molecule.

A dual approach has been used to determine a potential model for the neutral TTF molecule shown in Fig. 1. The program CLIMAX, ${ }^{13,14}$ developed to determine potential functions for molecules based on the intensity distribution for the normal modes in the INS spectrum, has been used to aid interpretation of the INS data. This analysis provides a potential model for both the in-plane and out-of-plane coordinates of the neutral TTF molecule. CLIMAX allows for the intensity contributions from phonon wings, overtones and combinations and for a range of instrumental factors and hence gives a reliable method for simulating INS spectra. The second approach has been to use Density Functional Theory (DFT) to provide a potential model for the ground electronic state of TTF and to use this potential to estimate the INS spectrum.

\section{EXPERIMENTAL AND COMPUTATIONAL DETAILS}

Tetrathiafulvalene (TTF) was used as obtained from Aldrich and stored at low temperature in the dark to avoid photodecomposition of the crystals. Inelastic neutron scattering data was collected on the Time Focused Crystal Analyzer (TFXA) using the pulsed neutron source, ISIS, at the Rutherford Appleton Laboratory, UK.

Raman spectra were measured at $10 \mathrm{~K}$ using a He cryostat coupled to a Jobin-Yvon U-1000 Raman spectrometer with CCD detection. Spectra-Physics model 2020 kryptonion and 2017 argon-ion lasers were used as the excitation source.

Energy calculations were performed using the Cerius2 implementation of DMOL3. ${ }^{15}$ A DND double numerical basis set was used with polarization functions for carbon. The radial extent of the integration mesh was 10 a.u. and the angular grid was adjusted to give numerical precision of 0.0001 a.u. Due to the number of calculations required, and the size of the system, the default functional (LDA, Perdew-Wang) was used. The vibrational spectra were calculated in the harmonic approximation employing the analytical first deriva-

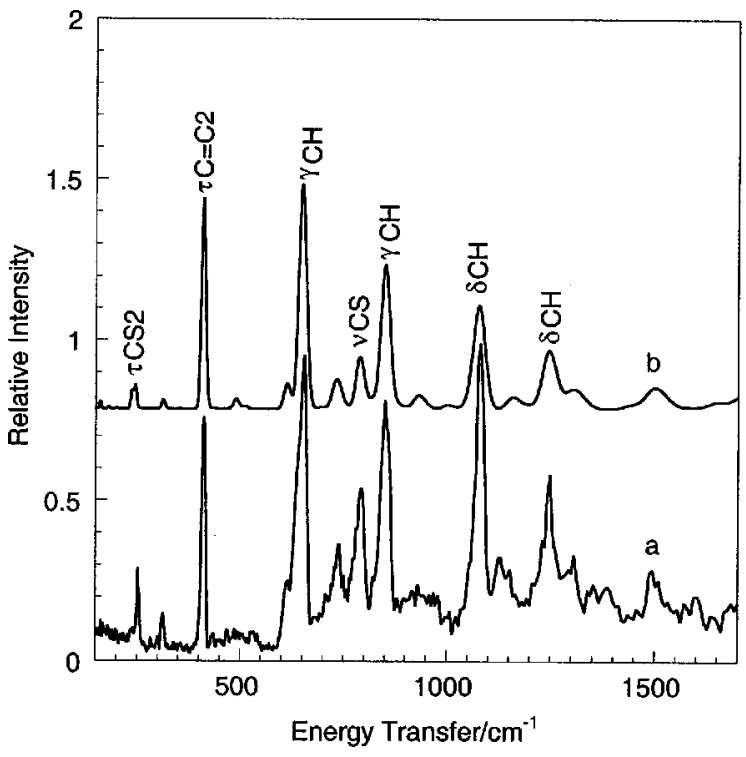

FIG. 2. The observed (a) and calculated (b) INS spectra for TTF based on the use of CLIMAX and showing assignments for the major peaks.

tives to obtain the forces. Starting from the equilibrium geometry each of the atoms was displaced along the three Cartesian directions. A simple energy calculation gives the Hellman-Feynmann forces on all the atoms from which the force constants are obtained by dividing by the displacement. The forces were obtained by making positive and negative displacements of $0.1 \AA$ and averaging the two force constants. The force constant matrix $F$ is then transformed to mass-dependent coordinates by the inverse kinetic energy matrix, $G$, giving the dynamical matrix. Diagonalization of the dynamical matrix gives the vibrational eigenvalues and eigenvectors.

The program CLIMAX was used to calculate the neutron spectral profile, from the normal modes, corresponding to the measured spectrum. The normal vibrational coordinates are

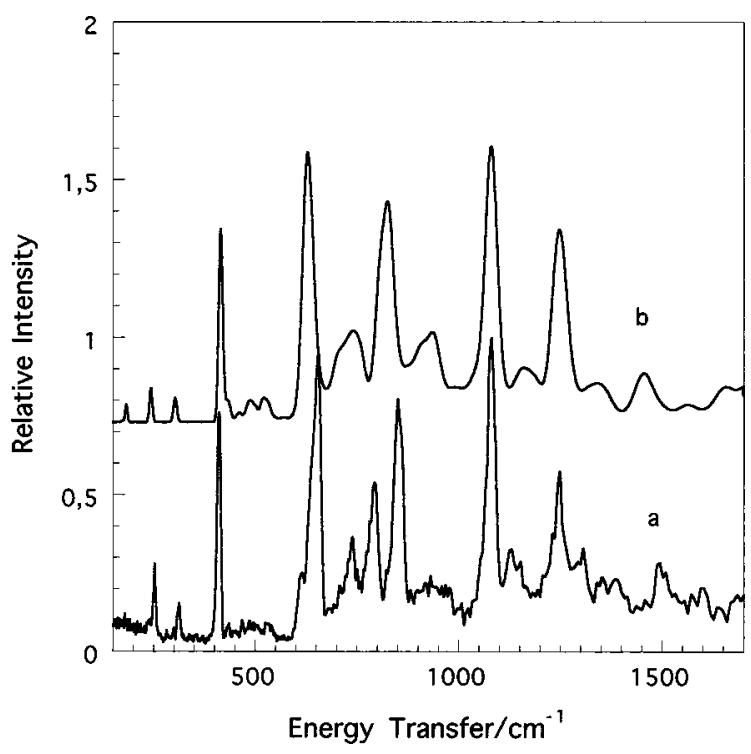

FIG. 3. The observed (a) and calculated (b) INS spectra for TTF based on the use of the DFT derived potential. 
TABLE I. Wave number data for TTF- $\mathrm{H}_{4}$ and TTF-D 4 based on the use of CLIMAX and for TTF- $\mathrm{H}_{4}$ from DMOL3.

\begin{tabular}{|c|c|c|c|c|c|c|c|c|}
\hline Obs. & $\begin{array}{c}\text { TTF- }_{4} \\
\text { DFT }\end{array}$ & Calc. & Obs.-Calc. & Obs. & $\begin{array}{c}\text { TTF-D }_{4} \\
\text { Calc. }\end{array}$ & Diff. & \multicolumn{2}{|c|}{ Assignment } \\
\hline 3100.0 & 3145 & 3100.0 & 0.0 & 2280.0 & 2296.0 & -16.0 & $a_{g}$ & $\nu \mathrm{ch}$ \\
\hline 1555.0 & 1596 & 1555.0 & 0.0 & 1544.0 & 1547.8 & -3.8 & $a_{g}$ & $\nu \mathrm{cc} 1$ \\
\hline 1516.0 & 1553 & 1516.0 & 0.0 & 1504.0 & 1495.1 & 8.9 & $a_{g}$ & $\nu \mathrm{cc} 2$ \\
\hline 1078.0 & 1080 & 1078.0 & 0.0 & 787.0 & 796.0 & -9.0 & $a_{g}$ & $\delta \mathrm{cch}+\delta \mathrm{sch}$ \\
\hline 736.0 & 763 & 736.0 & 0.0 & 715.0 & 715.7 & -0.7 & $a_{g}$ & $\nu \operatorname{cs} 2$ \\
\hline 467.0 & 484 & 467.0 & 0.0 & 470.0 & 467.0 & 3.0 & $a_{g}$ & $\nu \operatorname{cs} 1$ \\
\hline 237.0 & 243 & 237.0 & 0.0 & 242.0 & 234.0 & 8.0 & $a_{g}$ & $\delta \mathrm{scs}+\delta \csc$ \\
\hline 851.0 & 828 & 851.1 & -0.1 & & 717.0 & & $b_{1 g}^{\circ}$ & $\gamma \mathrm{ch}$ \\
\hline 410.0 & 417 & 408.0 & 2.0 & & 346.0 & & $b_{1 g}$ & $\pi \mathrm{cc} 2$ \\
\hline 650.0 & 629 & 656.5 & -6.5 & & 516.0 & & $b_{2 g}$ & $\gamma \mathrm{ch}$ \\
\hline 250.0 & 485 & 260.7 & -10.7 & & 261.0 & & $b_{2 g}$ & $\pi \mathrm{cs} 2$ \\
\hline 74.0 & 69 & 80.0 & -6.0 & & 75.0 & & $b_{2 g}$ & $\tau \mathrm{cs} 1$ \\
\hline 3050.0 & 3127 & 3050 & 0.0 & 2280.0 & 2254.0 & 26.0 & $b_{3 g}$ & $\nu \mathrm{ch}$ \\
\hline 1244.0 & 1245 & 1244.9 & -0.9 & 1057.0 & 1053.5 & 3.5 & $b_{3 g}$ & $\delta \mathrm{sch}+\delta \mathrm{cch}$ \\
\hline 998.0 & 994 & 997.9 & 0.1 & 975.0 & 977.0 & -2.0 & $b_{3 g}$ & $\delta \mathrm{cc} 1 \mathrm{~s}+\nu \mathrm{cs} 1$ \\
\hline 790.0 & 811 & 790.2 & -0.2 & 715.0 & 705.0 & 10.0 & $b_{3 g}$ & $\nu \operatorname{cs} 2$ \\
\hline 613.0 & 620 & 613.6 & -0.6 & 594.0 & 584.0 & 10.0 & $b_{3 g}$ & $\delta \csc$ \\
\hline 310.0 & 303 & 307.0 & 3.0 & 305.0 & 304.0 & 1.0 & $b_{3 g}$ & $\delta \mathrm{cc} 1 \mathrm{~s}$ \\
\hline 851.0 & 828 & 851.2 & -0.2 & & 717.0 & & $a_{u}$ & $\gamma \mathrm{ch}$ \\
\hline 410.0 & 413 & 408.7 & 1.3 & & 347.0 & & $a_{u}$ & $\tau \operatorname{cc} 2+\tau \operatorname{cs} 2$ \\
\hline 74.0 & 78 & 75.7 & -1.7 & & 75.0 & & $a_{u}$ & $\pi \mathrm{cc} 1$ \\
\hline 3100.0 & 3145 & 3100.0 & 0.0 & 2320.0 & 2296.0 & 24.0 & $b_{1 u}$ & $\nu \mathrm{ch}$ \\
\hline 1528.0 & 1566 & 1528.0 & 0.0 & 1504.0 & 1456.0 & -48.0 & $b_{1 u}$ & $\nu \mathrm{cc} 2$ \\
\hline 1078.0 & 1079 & 1078.0 & 0.0 & 780.0 & 800.0 & -20.0 & $b_{1 u}$ & $\delta \mathrm{cch}+\delta \mathrm{sch}$ \\
\hline 790.0 & 794 & 790.1 & -0.1 & 758.0 & 785.0 & -27.0 & $b_{1 u}$ & $\nu \mathrm{cs} 1$ \\
\hline 736.0 & 758 & 736.0 & 0.0 & 719.0 & 716.9 & 2.1 & $b_{1 u}$ & $\nu \operatorname{cs} 2$ \\
\hline 433.0 & 436 & 433.0 & 0.0 & 436.0 & 432.2 & 3.8 & $b_{1 u}$ & $\delta \csc$ \\
\hline 3050.0 & 3127 & 3050.0 & 0.0 & 2273.0 & 2254.0 & 19.0 & $b_{2 u}$ & $\nu \mathrm{ch}$ \\
\hline 1244.0 & 1242 & 1244.2 & -0.2 & 1040.0 & 1040.0 & 0.0 & $b_{2 u}$ & $\delta$ sch \\
\hline 851.0 & 868 & 851.5 & -0.5 & 865.0 & 840.4 & 24.6 & $b_{2 u}$ & $\nu \mathrm{cs} 1$ \\
\hline 790.0 & 803 & 789.6 & 0.4 & 703.0 & 707.0 & -4.0 & $b_{2 u}$ & $\nu \operatorname{cs} 2$ \\
\hline 613.0 & 627 & 612.1 & 0.9 & 603.0 & 582.6 & 20.4 & $b_{2 u}$ & $\delta \csc$ \\
\hline 100.0 & 87 & 97.4 & 2.6 & 108.0 & 94.7 & 13.3 & $b_{2 u}$ & $\delta \mathrm{cc} 1 \mathrm{~s}$ \\
\hline 650.0 & 630 & 645.1 & 4.9 & 492.0 & 502.0 & -10.0 & $b_{3 u}$ & $\gamma \mathrm{ch}$ \\
\hline 250.0 & 183 & 244.6 & 5.4 & 246.0 & 241.7 & 4.3 & $b_{3 u}$ & $\pi \mathrm{cs} 2$ \\
\hline 45.0 & -78 & 27.1 & 17.9 & 28.0 & 25.2 & 2.8 & $b_{3 u}$ & $\tau \mathrm{cs} 1$ \\
\hline
\end{tabular}

mass-weighted, the scattering cross sections of the different atoms are taken into account, overtone and combination frequencies are included, and the recoil of molecules is incorporated by convoluting the calculated peaks with the measured phonon spectrum.

\section{RESULTS AND DISCUSSION}

Calculated INS spectra have been obtained $a$ priori using DFT and by the use of the program CLIMAX based on assignments from INS, Raman, and infrared data. The two independent approaches give calculated spectra (see Figs. 2 and 3) that are in good agreement with the experimental INS data.

A preliminary potential function for TTF was based on a modified valence force field determined by fitting to the room temperature wave number data for the $\mathrm{TTF}-\mathrm{H}_{4}$ and TTF-D $_{4}$ isotopes. The assignments used were the solution values of Bozio et al. ${ }^{11}$ and with the exception of the assignment of $530 \mathrm{~cm}^{-1}$ as a $B_{2 g}$ mode these were also the assignments used by Liu et al. ${ }^{12}$ We favor an assignment of the $B_{2 g}$ mode at $250 \mathrm{~cm}^{-1}$ based on the bands observed in this region in both the INS and Raman spectra.
The TTF molecule is assumed to be planar with $D_{2 h}$ point group symmetry. The molecular structure and coordinate definitions are shown in Fig. 1. In order to restrict the number of potential constants we have refined the four closely related pairs of symmetry species, $A_{g} / B_{1 u}$, $B_{2 g} / B_{2 u}, B_{1 g} / A_{u}$, and $B_{2 g} / B_{3 u}$. This amounts to ignoring the effect of small interaction terms in the force constant matrix. The calculated wave numbers for the in-plane and out-of-plane modes for the two isotopes are reported in Table I together with the results from the DFT calculation for TTF$\mathrm{H}_{4}$. As previously observed ${ }^{11}$ the in-plane force field for the ring modes, based on the data for the two isotopes, shows a strongly coupled system, with large off-diagonal symmetrised force constants. In particular, minimization can be achieved using slightly different sets of constants for the two different $\mathrm{C}-\mathrm{S}$ bonds and the various ring deformation modes. The normal mode description is similar for these different sets of force constants, with strong coupling of the two $A_{g} \mathrm{C}=\mathrm{C}$ modes in addition to the expected coupling of the in-plane ring $\mathrm{C}-\mathrm{S}$ and deformation coordinates. Table I also gives the force constant(s) making the major contribution to the potential energy distribution for each mode. 
TABLE II. Symmetry force constants for TTF based on the wave number and intensity data from INS spectra.

\begin{tabular}{|c|c|c|c|c|c|}
\hline$F$ & $A_{g} / B_{1 u}$ & $B_{3 g} / B_{2 u}$ & $F$ & $B_{1 u} / A_{u}$ & $B_{2 g} / B_{3 u}$ \\
\hline CS1 & 3.470 & 2.254 & $\gamma \mathrm{CH}$ & 0.2571 & 0.2733 \\
\hline $\mathrm{CS} 2$ & 3.731 & 2.118 & $\tau \mathrm{CS} 2$ & 0.0418 & 0.1201 \\
\hline $\mathrm{CC} 2$ & 5.993 & & $\tau \mathrm{CC} 2$ & 0.0191 & \\
\hline $\mathrm{CC} 1$ & 7.903 & & $\tau \mathrm{CC} 1$ & 0.0166 & \\
\hline $\mathrm{CH}$ & 5.231 & 5.069 & $\tau \mathrm{CS} 1$ & 0.0233 & 0.0203 \\
\hline$\delta \mathrm{CSC}$ & 0.800 & 0.889 & $\gamma \mathrm{CH} / \tau \mathrm{CS} 2$ & -0.0650 & -0.0875 \\
\hline \&SCC2 & 0.593 & 0.302 & $\gamma \mathrm{CH} / \tau \mathrm{CS} 1$ & 0.0236 & \\
\hline$\delta \mathrm{SCC} 1$ & 0.380 & 0.654 & $\pi \mathrm{CS} 2 / \tau \mathrm{CS} 1$ & -0.0020 & \\
\hline$\delta S C S$ & 1.156 & & & & \\
\hline$\delta \mathrm{SCH}$ & 0.526 & 0.563 & & & \\
\hline $8 \mathrm{CCH}$ & 0.480 & 0.286 & & & \\
\hline $\mathrm{CS} 1 / \mathrm{CS} 2$ & 0.608 & -1.323 & & & \\
\hline $\mathrm{CS} 1 / \mathrm{CC} 2$ & -0.451 & & & & \\
\hline $\mathrm{CS} 1 / \delta \mathrm{SCC} 1$ & & 0.326 & & & \\
\hline $\mathrm{CS} 1 / \delta \mathrm{SCC} 2$ & 0.026 & -0.878 & & & \\
\hline $\mathrm{CS} 1 / \delta \mathrm{SCH}$ & & 0.100 & & & \\
\hline $\mathrm{CS} 1 / \delta \mathrm{CCH}$ & & 0.100 & & & \\
\hline $\mathrm{CS} 1 / \delta \mathrm{CSC}$ & & 0.300 & & & \\
\hline $\mathrm{CS} 1 / \delta \mathrm{SCS}$ & -0.028 & & & & \\
\hline $\mathrm{CS} 2 / \mathrm{CC} 2$ & 0.039 & & & & \\
\hline $\mathrm{CC} 2 / \delta \mathrm{CSC}$ & -0.150 & -1.598 & & & \\
\hline $\mathrm{CC} 2 / \delta \mathrm{SCC} 2$ & 0.117 & & & & \\
\hline $\mathrm{CC} 2 / \delta \mathrm{SCC} 1$ & 0.583 & & & & \\
\hline $\mathrm{CC} 2 / \delta \mathrm{SCS}$ & 0.238 & & & & \\
\hline $\mathrm{CC} 1 / \delta \mathrm{SCS}$ & -1.057 & & & & \\
\hline$\delta \mathrm{CSC} / \delta \mathrm{SCC} 2$ & -0.651 & & & & \\
\hline$\delta \mathrm{SCC} 2 / \delta \mathrm{SCH}$ & & -0.360 & & & \\
\hline$\delta \mathrm{SCC} 2 / \delta \mathrm{SCH}$ & & -0.360 & & & \\
\hline
\end{tabular}

The potential function obtained based on the room temperature infrared and Raman data was then used as the starting point to simulate the $20 \mathrm{~K}$ INS wave number and intensity data. The room temperature geometry has been assumed for TTF at $20 \mathrm{~K}$. This assumption is supported by the absence of any changes in the Raman spectrum of the monoclinic phase as the temperature is lowered to $20 \mathrm{~K}$ apart from the expected hardening of the phonons.

The calculated and observed INS spectra for TTF based on this data are shown in Fig. 2 and the potential parameters derived from the use of CLIMAX are reported in Table II. The assignments shown in Fig. 2 demonstrate the dominant contribution of the deformation modes involving protons and in particular the out-of-plane deformation modes. A good fit is achieved for the observed INS wave number and intensity data. The small differences for the observed and calculated intensities of the in-plane and out-of-plane modes can be explained in terms of the approximation of a planar $D_{2 h}$ TTF molecule in the crystalline state. The bent structure of $C_{2 v}$ symmetry would allow for coupling between the in-plane $A_{g}$ and out-of-plane $B_{3 u}$ symmetry species and some transfer of intensity between in-plane and out-of-plane modes. A similar intensity transfer is allowed by coupling between the $B_{3 g}$ and $A_{u}, B_{1 u}$ and $B_{2 g}$ and $B_{2 u}$ and $B_{1 g}$ pairs of $D_{2 h}$ symmetry. Evidence for a nonplanar geometry is apparent in the Raman spectrum of TTF where many infrared-active $u$ modes under $D_{2 h}$ point group symmetry are observed as weak bands. Figures 4 and 5 show the Raman bands in the region of the $\mathrm{CH}$ stretch and deformation modes and it is clear that factor

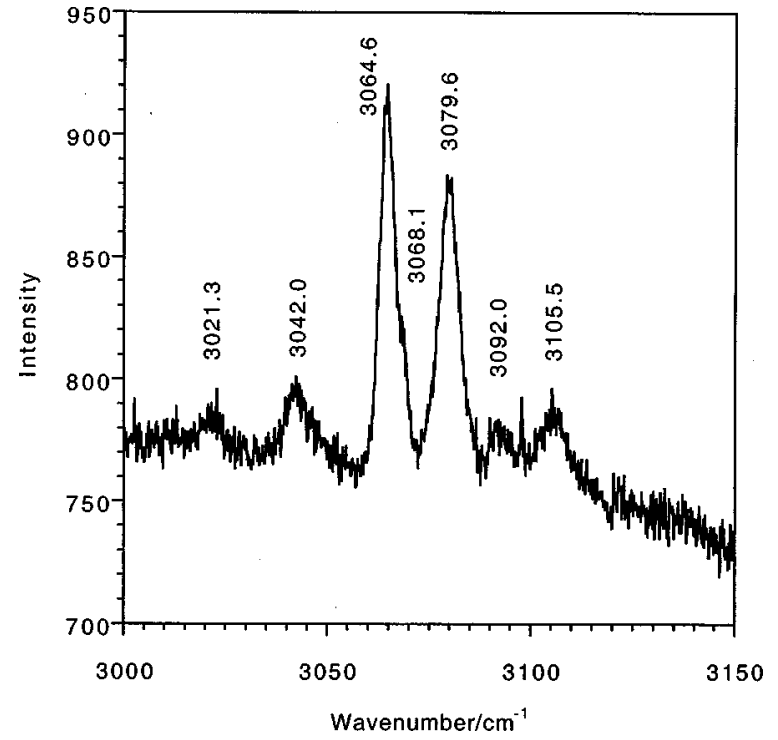

FIG. 4. The Raman bands observed for TTF in the $\mathrm{CH}$ stretch region, measured at $10 \mathrm{~K}$.

group components for both the $g$ and $u$ modes are present.

There remains some unexplained intensity in the INS spectrum at 1126, from 1352 to 1386 , and from 1573 to 1598 $\mathrm{cm}^{-1}$ (see Fig. 6), with a difference of approximately 250 $\mathrm{cm}^{-1}$ between successive bands. This intensity cannot be accounted for in terms of factor group splitting since the splitting observed at $8 \mathrm{~K}$ for the Raman-active phonons of TTF varies from 3 to $12 \mathrm{~cm}^{-1}$ in this region of the spectrum, as shown in Figs. 4 and 5 for the $\mathrm{CH}$ stretch and deformation bands. A possible explanation is that there is a transfer of momentum to one or more low wave number large amplitude modes, such as the $B_{2 u}$ doublet observed in the infrared at $244,250 \mathrm{~cm}^{-1}$. This mode has been assigned as the in-phase combination of the out-of-plane ring puckering of the two five-membered rings and is the mode most likely to be involved in the formation of the boat conformation reported

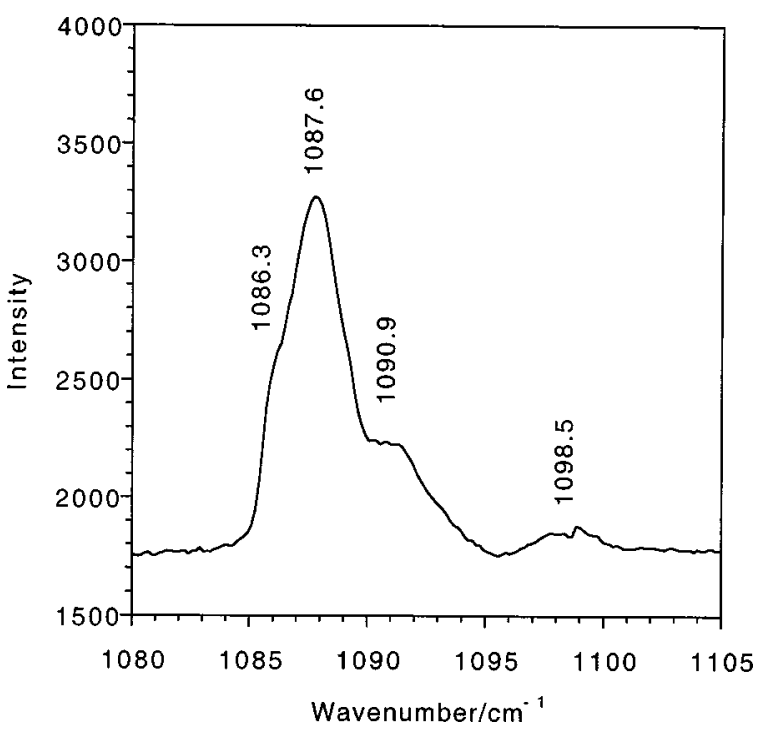

FIG. 5. The Raman bands observed for TTF in the $\mathrm{CH}$ in-plane deformation region, measured at $10 \mathrm{~K}$. 


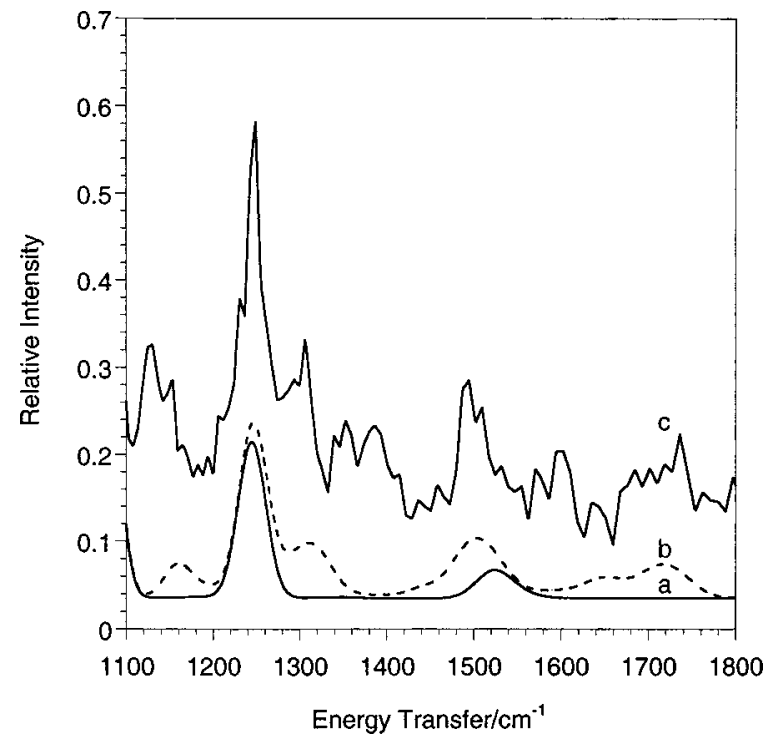

FIG. 6. The INS intensity attributable to overtone and combination bands in the region of the $\mathrm{C}=\mathrm{C}$ stretch modes. The spectrum with no overtones and combination bands (a) is compared with the spectrum including intensity from overtones and combinations (b) and with the observed INS intensity (c).

from NMR studies ${ }^{16}$ for TTF in nematic liquid crystalline solvents. However, there are a number of low wave number large amplitude modes and they may all be contributing additional phonon wing intensity.

The intensity calculated for the contribution from the phonon wings is either estimated based on an experimental Debye-Waller factor or if not known, the Debye-Waller factor is treated as a fitting parameter. In the case of TTF the Debye-Waller factor was used as a variable parameter and the estimated value indicates that there is reasonably strong coupling between the internal modes and some soft external modes. Notice in particular the high background intensity between 500 and $1500 \mathrm{~cm}^{-1}$ that is best explained as a contribution from phonon wings.

The other major contribution to the INS intensity, particularly in the region between 1000 and $2400 \mathrm{~cm}^{-1}$ is from overtone and combination bands. For example the intensity in the region where the $\mathrm{C}=\mathrm{C}$ modes might be expected to be observed is predominantly attributable to overtone and combination modes (see Fig. 6) and the two $A_{g}$ and the $B_{1 u}$ $\mathrm{C}=\mathrm{C}$ modes are almost invisible in the INS spectrum.

The a priori potential model based on the DFT calculation produces a remarkably close fit for the calculated compared to the observed intensities in the INS spectrum (Fig. 3 ). The assumption of a planar $D_{2 h}$ molecule may be the cause of the slight instability that results in negative phonon energy for one of the $B_{2 u}$ modes. DFT also estimates a relatively high wave number $\left(485 \mathrm{~cm}^{-1}\right)$ for a $B_{2 g}$ mode whereas we favor an assignment to the INS and Raman bands observed at approximately $250 \mathrm{~cm}^{-1}$. However, Bozio et al. favored an assignment for this band at $530 \mathrm{~cm}^{-1}$. We have been unsuccessful in obtaining a fit using the weak INS and Raman bands observed at approximately $490 \mathrm{~cm}^{-1}$. In the case of the INS data this intensity can be adequately accounted for in terms of contributions from overtone and combination modes. Additional arguments supporting our assignment are firstly that the energies of the $B_{3 u}$ and $B_{2 g}$ modes should be similar. If the band at $250 \mathrm{~cm}^{-1}$ assigned to a $B_{3 u}$ mode is correct, then it would be surprising if the corresponding $B_{2 g}$ mode were as high as $490 \mathrm{~cm}^{-1}$. Second, a different assignment for the $B_{3 u}$ mode would remove a major contribution to the INS intensity observed around 250 $\mathrm{cm}^{-1}$.

\section{CONCLUSIONS}

A good potential model has been derived for the in-plane and the out-of-plane modes of the neutral TTF molecule using DFT and based on low temperature Raman scattering and INS data.

This dual approach to the estimation of a potential model for TTF, based on the use of the CLIMAX code and on DFT, has led to a concordant set of force constants that explain the INS intensity for this relatively large molecule.

The INS spectrum of TTF is dominated by bands assigned to modes involving protons, and in particular to their out-of-plane modes. The spectral data demonstrates the advantage of INS spectroscopy for the determination of the vibrational energy states of modes involving protons.

The Raman data provides evidence for factor group splitting and the observation of weak scattering by the formally assigned $D_{2 h}$ infrared-active modes confirms a puckered geometry for the ground state of the neutral TTF molecule.

\section{ACKNOWLEDGMENTS}

The authors acknowledge support from the CNRS under the auspices of the Program International de Collaboration Scientifique. G.R.B. is grateful for the support of VUW for a period of research leave undertaken at Université Montpellier II.

${ }^{1}$ J. M. Williams, J. R. Ferraro, R. J. Thorn, K. D. Carlson, U. Geiser, H. H. Wang, A. M. Kini, and M.-H. Whangbo, Organic Superconductors (Prentice-Hall, New Jersey, 1992).

${ }^{2}$ E. Demiralp, S. Dasgupta, and W. A. Goddard III, J. Am. Chem. Soc. 117, 8154 (1995).

${ }^{3}$ A. Girlando, M. Masino, G. Visentini, A. Brillante, R. G. Della Valle, and E. Venuti, Synth. Met. 109, 13 (2000).

${ }^{4}$ J. S. Brooks, Adv. Mater. Opt. Electron. 8, 269 (1998).

${ }^{5}$ M. L. H. Green, Jingui Qin, D. O’Hare, H. E. Bunting, M. E. Thompson, S. R. Marder, and K. Chatakondu, Pure Appl. Chem. 61, 817 (1989).

${ }^{6}$ R. Backov, B. Bonnet, D. J. Jones, and J. Roziere, Chem. Mater. 9, 1812 (1997).

${ }^{7}$ T. Miyazaki, S. Matsuzaki, K. Ichimura, and M. Sano, Solid State Commun. 85, 949 (1993).

${ }^{8}$ M. R. Bryce, J. Mater. Chem. 10, 589 (2000).

${ }^{9}$ J. E. Eldridge, C. C. Homes, J. M. Williams, A. M. Kini, and H. H. Wang, Spectrochim. Acta, Part A 51A, 947 (1995).

${ }^{10}$ R. Bozio, A. Girlando, and D. Pecile, Chem. Phys. Lett. 52, 503 (1977).

${ }^{11}$ R. Bozio, I. Zanon, A. Girlando, and C. Pecile, J. Chem. Phys. 71, 2282 (1979).

${ }^{12}$ R. Liu, X. Zhou, and H. Kasmai, Spectrochim. Acta, Part A A53, 1241 (1997).

${ }^{13}$ G. J. Kearley, Nucl. Instrum. Methods Phys. Res. A 354, 53 (1995).

${ }^{14}$ G. J. Kearley, Spectrochim. Acta, Part A 48A, 349 (1992).

${ }^{15}$ B. Delley, J. Chem. Phys. 92, 508 (1990).

${ }^{16}$ T. C. Wong, E. E. Burnell, and L. Weiler, Chem. Phys. Lett. 50, 243 (1977). 